\title{
Association between $S T A T 3$ gene polymorphisms and ulcerative colitis susceptibility: a case-control study in the Chinese Han population
}

\author{
L. Wang ${ }^{1 *}$, Z-T. Wang ${ }^{1 *}$, H-X. Zhang ${ }^{2 *}$, J. Liu ${ }^{3}$, S-Y. Lu ${ }^{2}$, \\ R. Fan ${ }^{1}$, J. Zhou ${ }^{1}$, L. Xia ${ }^{1}$, Y-W. Sun ${ }^{1}$, J. Zhong ${ }^{1}$, and Y-Z. Yuan ${ }^{1}$ \\ ${ }^{1}$ Department of Gastroenterology, Ruijin Hospital, \\ Shanghai Jiaotong University School of Medicine, Shanghai, China \\ ${ }^{2}$ State Key Laboratory of Medical Genomics, \\ Research Center for Experimental Medicine, Ruijin Hospital, \\ Shanghai Jiaotong University School of Medicine, Shanghai, China \\ ${ }^{3}$ Shanghai Key Laboratory for Prevention and Treatment of Bone and Joint \\ Diseases with Integrated Chinese-Western Medicine, \\ Department of Orthopedics, \\ Shanghai Institute of Orthopedics and Traumatology, Ruijin Hospital, \\ Shanghai Jiaotong University School of Medicine, Shanghai, China \\ *These authors contributed equally to this study. \\ Corresponding author: J. Zhong \\ E-mail: Jimmyzj64@medmail.com.cn
}

Genet. Mol. Res. 13 (2): 2343-2348 (2014)

Received March 30, 2013

Accepted June 7, 2013

Published April 3, 2014

DOI http://dx.doi.org/10.4238/2014.April.3.6

\begin{abstract}
Ulcerative colitis (UC) is a chronic inflammation of the large intestine. The aim of this study was to investigate the association of two polymorphisms in STAT3 with the risk of UC development in the Chinese Han population. This is a hospital-based case-control study involving $56 \mathrm{UC}$ patients and 274 controls. Genotyping was performed using the polymerase chain reaction with sequence-specific primers (PCR-
\end{abstract}


SSP) method. Statistical analyses were conducted using logistic regression and genotype risk score. Overall, there was a significant difference between patients and controls in the genotype distribution of rs2293152 $(\mathrm{P}=0.044)$. The risk for UC associated with the rs $2293152-\mathrm{G}$ mutant allele was increased (odds ratio $=2.76 ; 95 \%$ confidence interval $=1.06$ 7.24) under the dominant model. However, we failed to find any obvious differences in the rs 4796793 genotype or allele distributions between the UC patients and controls, and did not detect any significant association of the rs4796793 polymorphism with UC across different genetic models of inheritance. Our study implies that the STAT3 rs2293152 polymorphism may be associated with the occurrence of UC and might be used as a predictive factor for $\mathrm{UC}$ in the Chinese Han population.

Key words: STAT3 gene; Ulcerative colitis; Polymorphism

\section{INTRODUCTION}

Ulcerative colitis (UC) is a chronic inflammation of the large intestine that, together with Crohn's disease (CD), makes up the majority of what are called inflammatory bowel diseases (IBD). The etiology and pathogenesis of UC are currently not fully understood. However, familial aggregation and twin studies reported that patients with UC carry a strong genetic predisposition (Zheng et al., 2003). Moreover, dozens of studies strongly suggest that UC results from a combination of factors, such as commensal bacteria, food antigens, and immunologic factors, as well as multiple genetic factors (Molodecky and Kaplan, 2010; Cho, 2008).

The signal transducer and activator of transcription 3 (STAT3) is a member of a family of seven proteins (STATs 1, 2, 3, 4, 5a, 5b, and 6), which plays an important role in the development of the human immune system and hematopoiesis. It has been implicated in the signal transduction pathways of multiple cytokines, including the IL-2/ $\gamma \mathrm{c}$, IL-6/gp130, IFN, and IL-10 families of cytokines, as well as IL-12, IL- 23, Flt3 ligand, M-CSF, G-CSF, leptin, and growth hormone (Takeda et al., 1999; Akira, 2000; Laouar et al., 2003; Murray, 2007; Stumhofer et al., 2007; O'Shea and Murray, 2008). Several studies have highlighted that the STAT3 signaling pathway is important in the occurrence and development of IBD in both patient and animal models (Suzuki et al., 2001; Lovato et al., 2003; Musso et al., 2005; Li et al., 2012). In a genome-wide association study, Barrett et al. (2008) reported that the STAT3 locus is significantly associated with susceptibility to $\mathrm{CD}$. Since then, a number of studies demonstrated that STAT3 polymorphisms are associated with $\mathrm{UC}$ as well as $\mathrm{CD}$, but the results were not consistent in different population cohorts (Franke et al., 2008; Sato et al., 2009; Cenit et al., 2010; Ferguson et al., 2010; Peter et al., 2011; Polgar et al., 2012). Therefore, in this study, we analyzed the association between UC and two STAT3 polymorphisms (rs2293152 and rs4796793) in the Chinese Han population.

\section{MATERIAL AND METHODS}

\section{Patient and control subjects}

This was a hospital-based case-control study of the Chinese Han population performed 
between January 2009 and December 2010. It involved 56 UC patients and 274 healthy controls recruited from the Gastroenterology Department of Ruijin Hospital appended to Shanghai Jiaotong University School of Medicine. All patient diagnoses were made by senior physicians and were based on clinical, endoscopic, radiological, and histopathological findings, in accordance with previously established international criteria (Ooi et al., 2010). All patients were registered with an integrated clinical and epidemiological registry and were followed up for at least one year. Controls were randomly selected from healthy individuals receiving routine health screening. The study was approved by the Research Ethics Committee of Ruijin Hospital, Shanghai, China. Informed consent was obtained from all subjects before blood sampling.

\section{Genotyping}

Genomic DNA was isolated from EDTA peripheral blood using QIAamp blood extraction kit (Qiagen, Hilden, Germany) following manufacturer instructions. All DNA samples were genotyped for single nucleotide polymorphisms (SNPs) by polymerase chain reaction with sequence-specific primers (PCR-SSP). All primers for PCR-SSP were designed using genomic sequences in GenBank (http://www.ncbi.nlm.nih.gov). The primer sequences are listed in Table 1. The amplified products were assessed for the presence/absence of PCR amplicons specific to the particular alleles using standard $2 \%$ agarose gel electrophoresis followed by ethidiumbromide staining. About $10 \%$ of the samples were then confirmed by DNA sequencing.

\begin{tabular}{lll}
\multicolumn{2}{l}{ Table 1. Primer sequences used for genotyping. } & \\
\hline Polymorphisms & Primer & Sequence \\
\hline rs 2293152 & Internal control forward primer & CCGTTTAACCTAACTTCAT \\
& Common reverse primer & CCAGTTGTCTTCATCCC \\
& Specific primer C & ACAAAGGGCCTCTGGCTGCC \\
rs4796793 & Specific primer G & ACAAAGGGCCTCTGGCTGCG \\
& Internal control forward primer & TCTGGTAGACACAGCTCAGTATGG \\
& Common reverse primer & CCATAGTCGCAGAGGTAGATTTA \\
& Specific primer C & TGTTTAGTGATTTACTGCTTACAAAGG \\
& Specific primer G & TGTTTAGTGATTTACTGCTTACAAAGC \\
\hline
\end{tabular}

\section{Statistical analysis}

Comparisons between UC patients and controls were conducted by the unpaired $t$-test for continuous variables, and by the $\chi^{2}$ test for categorical variables. To avoid gross genotyping error, all polymorphisms were checked for consistency with Hardy-Weinberg equilibrium on a contingency table of observed-versus-predicted genotype frequencies, using Pearson's $\chi^{2}$ test or Fisher's exact test. Genotypes were compared by logistic regression analysis, assuming additive, dominant, and recessive models of inheritance, respectively. Statistical significance was defined as $\mathrm{P}<0.05$.

\section{RESULTS}

Detailed information on patients and controls is shown in Table 2. Cases and controls were well matched by age and gender distribution. The frequency and distribution of alleles and genotypes of the rs2293152 and rs4796793 polymorphisms in STAT3 were identified and 
compared between UC patients and controls. The genotype distributions of the two polymorphisms were in Hardy-Weinberg equilibrium in the control groups $(\mathrm{P}>0.05)$.

Table 2. Characteristics of ulcerative colitis (UC) patients and healthy controls in the Chinese Han population.

\begin{tabular}{lcc}
\hline Characteristics & UC patients & Control subjects \\
\hline Number & 56 & 274 \\
Age (mean \pm SD, years) & $42.5 \pm 16.7$ & $50.3 \pm 12.5$ \\
Age range (years) & $3-77$ & $18-70$ \\
Male/female & $33 / 23$ & $155 / 119$ \\
\hline
\end{tabular}

As shown in Table 3, a significant difference between UC patients and controls was observed in genotype distribution for rs2293152, but not in allele distribution ( $\mathrm{P}^{\text {allele }}=0.399$, and $\mathrm{P}^{\text {genotype }}=0.044$ ). A significant difference was identified for $\mathrm{rs} 2293152$ in association with $\mathrm{UC}$ under the dominant model $(\mathrm{OR}=2.76 ; 95 \% \mathrm{CI}=1.06-7.24)$, while no significant association was detected under the additive $(\mathrm{OR}=1.21 ; 95 \% \mathrm{CI}=0.79-1.85)$ or recessive $(\mathrm{OR}=0.83$; $95 \% \mathrm{CI}=0.43-1.60)$ models.

Table 3. Genotype distributions and allele frequencies of the polymorphisms studied between patients and controls, and their risk prediction for UC under three genetic models of inheritance.

\begin{tabular}{|c|c|c|c|c|c|}
\hline Polymorphism & Patients $(\mathrm{N}=56)$ & Controls $(\mathrm{N}=274)$ & $\mathrm{P} \chi^{2}$ & Genetic models & OR $(95 \% \mathrm{CI})$ \\
\hline \multicolumn{6}{|l|}{ rs2293152 } \\
\hline $\mathrm{CC}$ & 5 & 58 & & Additive & $1.21(0.79-1.85)$ \\
\hline $\mathrm{CG}$ & 37 & 136 & 0.044 & Dominant & $2.76(1.06-7.24)$ \\
\hline GG & 14 & 78 & & Recessive & $0.83(0.43-1.60)$ \\
\hline $\mathrm{C}(\%)$ & 42.0 & 46.3 & 0.399 & & \\
\hline \multicolumn{6}{|l|}{ rs4796793 } \\
\hline $\mathrm{CC}$ & 23 & 112 & & Additive & $1.08(0.72-1.62)$ \\
\hline CG & 23 & 121 & 0.776 & Dominant & $1.00(0.56-1.80)$ \\
\hline GG & 10 & 39 & & Recessive & $1.30(0.61-2.79)$ \\
\hline $\mathrm{C}(\%)$ & 61.6 & 63.4 & 0.717 & & \\
\hline
\end{tabular}

With regard to rs4796793, there was no significant difference in genotype or allele distributions between UC patients and controls, and this non-significance was mirrored under the assumptions of the additive $(\mathrm{OR}=1.08 ; 95 \% \mathrm{CI}=0.72-1.62)$, dominant $(\mathrm{OR}=1.00$; $95 \% \mathrm{CI}=0.56-1.80)$, and recessive $(\mathrm{OR}=1.30 ; 95 \% \mathrm{CI}=0.61-2.79)$ models.

\section{DISCUSSION}

The STAT3 gene is located on chromosome 17q21. Its protein product is a member of the STAT protein family that carries out a dual function: signal transduction and activation of transcription. STAT3 is a widely expressed latent cytoplasmic transcription factor that relays signals from the cell membrane directly to the nucleus. STAT3 becomes activated as a DNA binding protein through phosphorylation of tyrosine in response to a variety of stimuli and mediates the expression of a variety of genes. Thus, it plays a key role in many biological pathways crucial to cell function, including proliferation, migration, survival, and differentiation (Levy and Darnell, 2002). Several studies indicate that STAT3 activation plays distinctly different roles in innate and acquired immune responses in colitis: activation of STAT3 in innate immune cells enhances mucosal barrier function, whereas STAT3 activation in T-cells exacerbates colitis (Lovato et al., 2003; Musso et al., 2005). A number of studies also suggest 
that polymorphisms of STAT3 are associated with susceptibility to CD or UC in some population cohorts (Franke et al., 2008; Sato et al., 2009; Cenit et al., 2010; Ferguson et al., 2010; Peter et al., 2011; Polgar et al., 2012).

In this study, we examined the rs2293152 and rs4796793 polymorphisms of STAT3 in $56 \mathrm{UC}$ patients and 274 normal controls from the Chinese Han population. rs2293152, which has been reported to be significantly associated with CD in the Japanese population (Sato et al., 2009), was associated with susceptibility to UC in the Chinese Han population in this study. rs4796793, which has been reported to be associated with dilated cardiomyopathy in the Chinese Han population (Peng et al., 2012), did not show significant association with UC when studied in the UC patient and normal control groups.

A general problem with multifactorial disorders like UC is that the association of one gene with the disease in one population cannot be exactly replicated in another population. Since the incidence, epidemiology and phenotype are different between patients from the Chinese Han population and western countries, the genetic susceptibility may be different too. Other reasons, such as sample size and different endophenotypes, may also lead to inconsistency. In this study, our sample size was not large, so more SNP sites for pair-loci D' $/ \mathrm{r}^{2}$ value and haplotype analyses are necessary on a larger number of Chinese subjects and on other ethnicities to confirm the association. Nevertheless, our study is the first demonstration of the single-marker association of STAT3 with UC susceptibility in the Chinese Han population. Our results provide a reference for further studies on the STAT3 gene in other populations.

\title{
ACKNOWLEDGMENTS
}

\author{
(\#31000408).
}

\section{REFERENCES}

Akira S (2000). Roles of STAT3 defined by tissue-specific gene targeting. Oncogene 19: 2607-2611.

Barrett JC, Hansoul S, Nicolae DL, Cho JH, et al. (2008). Genome-wide association defines more than 30 distinct susceptibility loci for Crohn's disease. Nat. Genet. 40: 955-962.

Cenit MC, Alcina A, Marquez A, Mendoza JL, et al. (2010). STAT3 locus in inflammatory bowel disease and multiple sclerosis susceptibility. Genes Immun. 11: 264-268.

Cho JH (2008). The genetics and immunopathogenesis of inflammatory bowel disease. Nat. Rev. Immunol. 8: 458-466.

Ferguson LR, Han DY, Fraser AG, Huebner C, et al. (2010). Genetic factors in chronic inflammation: single nucleotide polymorphisms in the STAT-JAK pathway, susceptibility to DNA damage and Crohn's disease in a New Zealand population. Mutat. Res. 690: 108-115.

Franke A, Balschun T, Karlsen TH, Hedderich J, et al. (2008). Replication of signals from recent studies of Crohn's disease identifies previously unknown disease loci for ulcerative colitis. Nat. Genet. 40: 713-715.

Levy DE and Darnell JE Jr (2002). Stats: transcriptional control and biological impact. Nat. Rev. Mol. Cell Biol. 3: 651662.

Li Y, de Haar C, Peppelenbosch MP and van der Woude CJ (2012). New insights into the role of STAT3 in IBD. Inflamm. Bowel. Dis. 18: 1177-1183.

Laouar Y, Welle T, Fu XY and Flavell RA (2003). STAT3 is required for flt3L-dependent dendritic cell differentiation. Immunity 19: 903-912.

Lovato P, Brender C, Agnholt J, Kelsen J, et al. (2003). Constitutive STAT3 activation in intestinal T cells from patients with Crohn's disease. J. Biol. Chem. 278: 16777-16781.

Molodecky NA and Kaplan GG (2010). Environmental risk factors for inflammatory bowel disease. Gastroenterol. Hepatol. 6: 339-346. 
Murray PJ (2007). The JAK-STAT signaling pathway: input and output integration. J. Immunol. 178: 2623-2629.

Musso A, Dentelli P, Carlino A, Chiusa L, et al. (2005). Signal transducers and activators of transcription 3 signaling pathway: an essential mediator of inflammatory bowel disease and other forms of intestinal inflammation. Inflamm. Bowel Dis. 11: 91-98.

O’Shea JJ and Murray PJ (2008). Cytokine signaling modules in inflammatory responses. Immunity 28: 477-487.

Ooi CJ, Fock KM, Makharia GK, Goh KL, et al. (2010). The Asia-Pacific consensus on ulcerative colitis. J. Gastroenterol. Hepatol. 25: 453-468.

Peng Y, Zhou B, Wang Y, Chen Y, et al. (2012). Association between polymorphisms in the signal transducer and activator of transcription and dilated cardiomyopathy in the Chinese Han population. Mol. Cell Biochem. 360: 197-203.

Peter I, Mitchell AA, Ozelius L, Erazo M, et al. (2011). Evaluation of 22 genetic variants with Crohn's disease risk in the Ashkenazi Jewish population: a case-control study. BMC Med. Genet. 12: 63.

Polgar N, Csongei V, Szabo M, Zambo V, et al. (2012). Investigation of JAK2, STAT3 and CCR6 polymorphisms and their gene-gene interactions in inflammatory bowel disease. Int. J. Immunogenet. 39: 247-252.

Sato K, Shiota M, Fukuda S, Iwamoto E, et al. (2009). Strong evidence of a combination polymorphism of the tyrosine kinase 2 gene and the signal transducer and activator of transcription 3 gene as a DNA-based biomarker for susceptibility to Crohn's disease in the Japanese population. J. Clin. Immunol. 29: 815-825.

Stumhofer JS, Silver JS, Laurence A, Porrett PM, et al. (2007). Interleukins 27 and 6 induce STAT3-mediated T cell production of interleukin 10. Nat. Immunol. 8: 1363-1371.

Suzuki A, Hanada T, Mitsuyama K, Yoshida T, et al. (2001). CIS3/SOCS3/SSI3 plays a negative regulatory role in STAT3 activation and intestinal inflammation. J. Exp. Med. 193: 471-481.

Takeda K, Clausen BE, Kaisho T, Tsujimura T, et al. (1999). Enhanced Th1 activity and development of chronic enterocolitis in mice devoid of Stat3 in macrophages and neutrophils. Immunity 10: 39-49.

Zheng CQ, Hu GZ, Zeng ZS, Lin LJ, et al. (2003). Progress in searching for susceptibility gene for inflammatory bowel disease by positional cloning. World J. Gastroenterol. 9: 1646-1656. 